\title{
Successful peroral endoscopic myotomy performed in Endoscopy Department as a radical, long-term treatment for esophageal achalasia - the Greek experience
}

\author{
This article was published in the following Dove Press journal: \\ Therapeutics and Clinical Risk Management \\ 14 February 2017 \\ Number of times this article has been viewed
}

\section{Nikolas Eleftheriadis ${ }^{1,2}$ \\ Eleni Damianos \\ Eleftheriadou ${ }^{2}$}

'Endoscopy Department,

Gastroenterology Unit, Metropolitan Hospital Athens, ${ }^{2}$ Medical School, Aristotle University of Thessaloniki, Thessaloniki, Greece

Video abstract

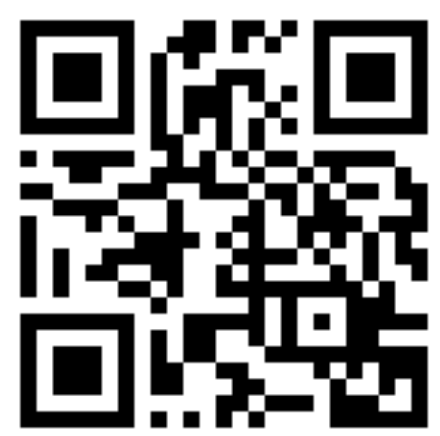

Point your SmartPhone at the code above. If you have a QR code reader the video abstract will appear. Or use:

http://youtu.be/8g5jW8h04N

Correspondence: Nikolas Eleftheriadis Endoscopy Department,

Gastroenterology Unit, Metropolitan Hospital Athens, Politechniu 3 Triandria, Thessaloniki-PC 55337, Greece

Tel +30 23I I283002

Email nikoseleftheriadis@yahoo.com
Introduction: Peroral endoscopic myotomy (POEM) has been considered as a minimalinvasive, innovative technique for long-term treatment of all types of esophageal achalasia and other esophageal motility disorders.

Patients and methods: We report on 20 consecutive Greek patients with manometrically proved esophageal achalasia (14 patients with type I, 4 with type II, 2 with type III, and 4 with sigmoid esophagus), with an age range of 32-92 years, mean age 59 years, 12 males, successfully treated by POEM from 2013 to 2015. The Eckardt score was 7-12 (type III). Seventeen (85\%) POEM procedures were performed in the Endoscopy Department, according to a previous study. During POEM, $\mathrm{CO}_{2}$ insufflation was mandatory, while the Triangle Tip knife was the only knife used in all procedures. Eckardt score, esophagogram and manometry before and after performing POEM were used for evaluation of our results. The follow-up period was 6 months to 3 years.

Results: Selective circular myotomy, $10-13 \mathrm{~cm}$ in length, was successfully completed in all patients without severe acute or late complications. Three patients $(15 \%)$ showed moderate pneumomediastinum and pneumoperitoneum, which was successfully managed by abdominal needle drainage during the procedure. One patient showed mild pleural collection, and in one patient the clip-endoloop technique was used to successfully close the mucosal entry after the completion of POEM. The outcome was uneventful without any further clinical consequences. No other short- or long-term serious complications were reported. Patients were discharged after 1-3 days of hospitalization. Six months to 3 years after the POEM procedure, all patients were alive; the majority (90\%) had complete clinical improvement, while two patients with sigmoidtype achalasia showed moderate-to-significant clinical improvement. Erosive esophagitis was reported in $15 \%$.

Conclusion: Our results are in accordance with international data, and proved the safety and efficacy of the POEM technique for radical long-term treatment of all types of achalasia, including end-stage sigmoid-type achalasia, in the Endoscopy Department. However, long-term follow-up is necessary and awaited.

Keywords: achalasia, Heller myotomy, peroral endoscopic myotomy

\section{Introduction}

Peroral endoscopic myotomy (POEM) ${ }^{1}$ has been considered the most modern, innovative, minimal-invasive, long-term treatment not only for all types of esophageal achalasia (classical [I], vigorous [II] or spastic [III] according to the Chicago ${ }^{2}$ classification) but also for other esophageal motility disorders (diffuse esophageal spasm, nutcracker and 
jackhammer esophagus). ${ }^{3}$ Furthermore, this technique has been extended and applied for sigmoid-type achalasia ${ }^{4,5}$ in failure of previous surgical or laparoscopic Heller myotomy $(\mathrm{LHM})^{6,7}$ and also in failure of previous POEM. ${ }^{4}$

After the first successful POEM procedure in humans by Inoue et $\mathrm{al}^{1}$ in 2008 , the POEM technique has been spread worldwide and is currently performed in multiple International centers, ${ }^{8}$ and in Greece. ${ }^{5,8}$

The aim of the present study was to report our experience from the POEM procedure in Greek patients with achalasia.

\section{Patients and methods}

We report the safe and successful application of the POEM procedure in 20 consecutive Greek patients with manometrically proved esophageal achalasia, with age range 32-92 years, mean age 59 years, 12 males, 17 of them treated in the Endoscopy Department from 2013 to 2015. According to the Chicago ${ }^{2}$ classification, 14 patients were classified as type I, 4 as type II, 2 as type III, and 4 patients with sigmoid esophagus. The Eckardt score was 7-12 (type III). Seventeen (85\%) POEM procedures were performed in the Endoscopy Department, under general anesthesia with intubation of the patients in the supine position, according to the previous description. ${ }^{1,9}$

The study received approval from the institutional review board of Metropolitan Hospital Athens Greece. A written informed consent was also obtained from all patients.
All patients were placed on a clear liquid diet for 24 hours before performing the procedure with fasting from previous afternoon, then were admitted to the hospital the morning of the operation, and after routine preoperative examinations, POEM was performed. Endoscopic clearance of esophageal contents was routinely performed before POEM, while in patients with sigmoid-type achalasia, endoscopic clearance of residues was performed on the day before POEM to avoid aspiration during induction of anesthesia. Flushing with water containing small amounts of simethicone was routinely done to eliminate gas and foamy mucus in esophagus.

Treatment with prophylactic intravenous antibiotics, usually cefuroxime $750 \mathrm{mg}$ IV three times per day, was started during POEM and continued until the discharge in uncomplicated cases. Then patients were administered an additional 4 days of oral antibiotics. Also, $80 \mathrm{mg}$ of gentamicin diluted with $20 \mathrm{~mL}$ of saline was routinely sprayed into the submucosal tunnel before mucosal closure, according to the initial description by Inoue et al. ${ }^{1}$ No other specific measures were followed.

POEM started with a mucosal incision, followed by submucosal tunnel creation crossing the esophagogastric junction (EGJ) and myotomy. Finally, the mucosal entry was closed with endoscopic clip placement (Figures 1 and 2).

A forward-viewing high-resolution gastroscope with a transparent distal tapered-type (Fujinon) cap attached to the tip of the endoscope was used, with water jet for irrigation, while carbon dioxide $\left(\mathrm{CO}_{2}\right.$; Olympus Medical Systems, Tokyo,
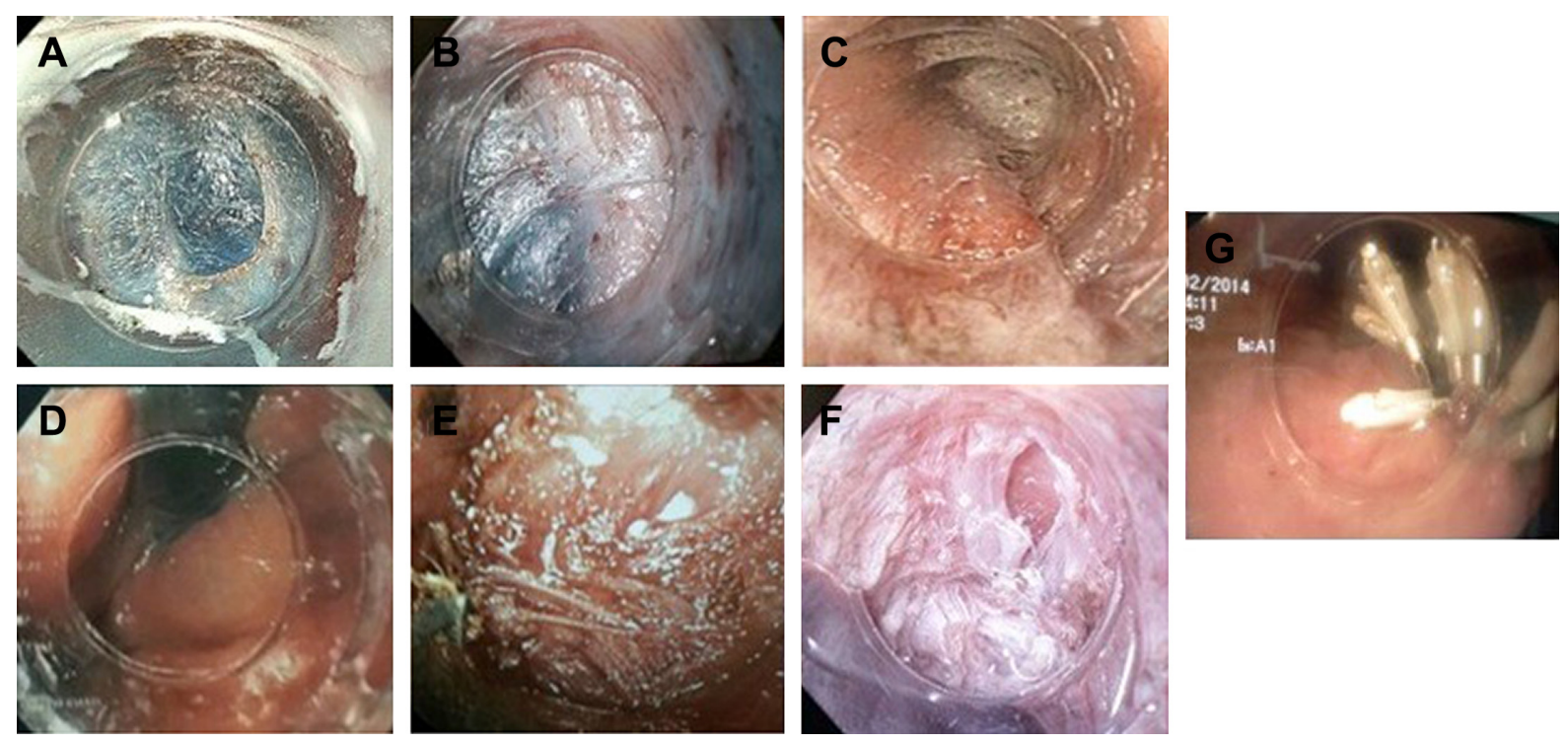

Figure I Stages of anterior POEM: (A) longitudinal mucosal incision at the second hour. (B) Submucosal tunnel. (C) Identification of EGJ inside the tunnel (palisade vessels). (D) Blue dye is recognized at retroversion in the gastric cardia. (E) Selective circular myotomy is performed using TT knife. (F) External longitudinal muscle layer is recognized at the back of the myotomy side. Longitudinal muscle fibers split each other. (G) Mucosal entry closing by clips at the completion of myotomy. Abbreviations: EGJ, esophagogastric junction; TT, Triangle Tip; POEM, peroral endoscopic myotomy. 

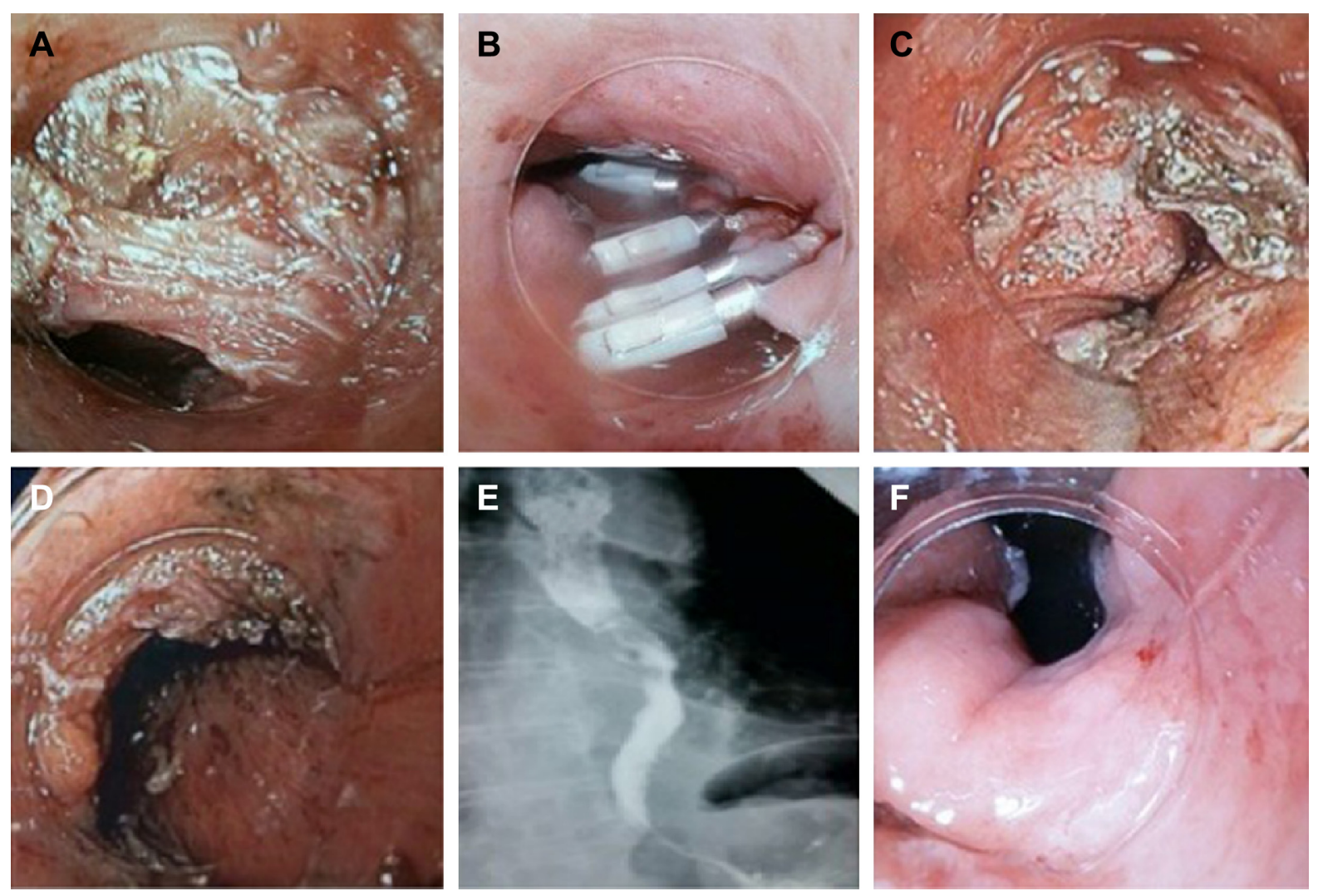

Figure 2 Successful bilateral POEM in a 92-year-old female with sigmoid-type-III achalasia and frequent episodes of pneumonic aspiration: (A) anterior myotomy. Circular muscle layer is relatively thick. (B) Mucosal entry closing by clips after anterior POEM. (C, D) Redo-posterior POEM. Myotomy site and mucosa are recognized within the submucosal tunnel. (E) Esophagogram I day after posterior POEM showed rapid passage of the contrast medium (gastrografin). (F) Easy passage of the endoscope without resistance through GEJ and open GEJ in retroversion after posterior POEM.

Abbreviations: GEJ, gastroesophageal junction; POEM, peroral endoscopic myotomy.

Japan) was obligatory for insufflation in all POEM procedures at all stages. Positive pressure ventilation with higher pressure than those generated by endoscopic $\mathrm{CO}_{2}$ insufflation was also used to reduce the risk of mediastinal emphysema. ${ }^{10}$

Triangle Tip knife was the only knife used at all steps for all POEM procedures. Eckardt score, esophagogram before and after POEM (Figure 3), and manometry, particularly
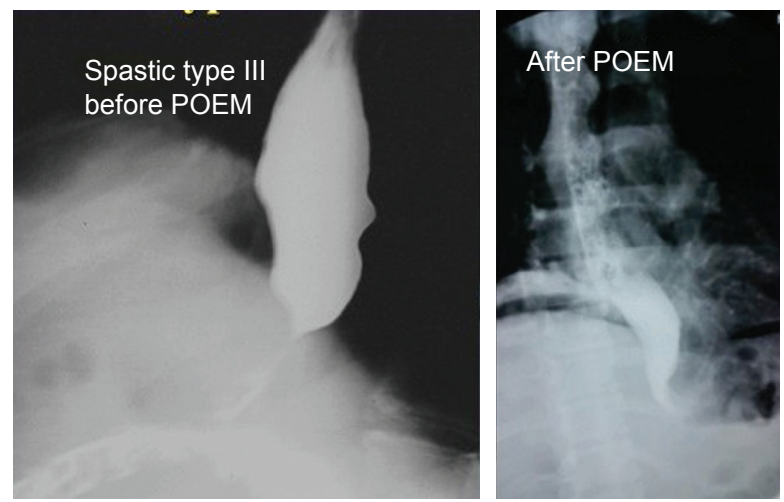

Figure 3 Esophagogram of a 56-year-old female with achalasia type III before and after anterior POEM. Rapid passage of the contrast medium after POEM. Abbreviation: POEM, peroral endoscopic myotomy. high-resolution topographic manometry ${ }^{4}$ (Figure 4), were used for the diagnosis of achalasia and evaluation of the efficacy of POEM. The follow-up period was 6 months to 3 years.

\section{Results}

All POEM procedures were successfully completed uneventfully, according to the international standards, and myotomies were effectively extended 2-3 $\mathrm{cm}$ below the EGJ. Seventeen $(17 / 20,85 \%)$ of POEM procedures were performed and completed in Endoscopy Department, without serious short- or long-term complications. The majority of the patients (13/20, $65 \%$ ) were discharged the next day after POEM; Also, the POEM technique was safely and successfully applied to octogenarians.

Selective circular myotomy, $10-13 \mathrm{~cm}$ in length, was successfully completed in all patients with extension of myotomy $2-3 \mathrm{~cm}$ below the gastroesophageal junction, as this was controlled by identification of blue dye at the gastric cardia at the end of the procedure.

Three patients (15\%) showed moderate pneumomediastinum and pneumoperitoneum, which was successfully 


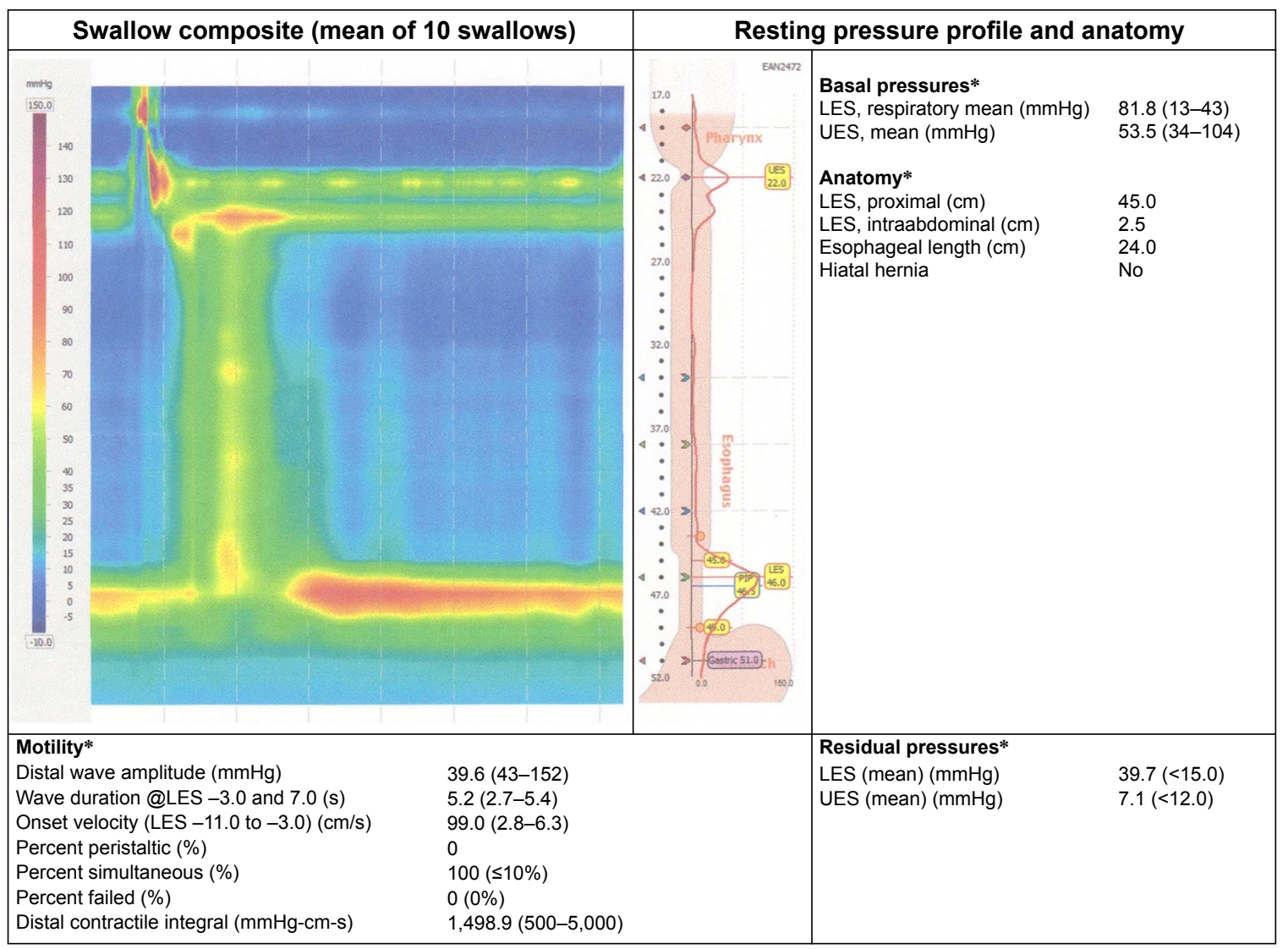

Figure 4 HRTM in a 70-year-old male with spastic type III achalasia, who underwent a successful POEM.

Notes: *Motility values are mean among swallows; Normal values of integrated relaxation pressure $<15.0 \mathrm{mmHg}$ and residual LES pressure normal $<15.0 \mathrm{mmHg}$. Simultaneous contractions: Velocity $>8.0 \mathrm{~cm} / \mathrm{s}$.

Abbreviations: HRTM, high-resolution topographic manometry; POEM, peroral endoscopic myotomy; LES, lower esophageal sphincter; USE, upper esophageal sphincter.

managed by abdominal needle drainage during the procedure. Actually pneumoperitoneum or pneumomediastinum should not be considered as complications but as normal consequence of POEM, in accordance with pneumomediastinum and pneumoperitoneum in thoracoscopic or laparoscopic procedures, respectively.

One patient showed mild asymptomatic pleural collection without clinical significance, and in one patient the clip-endoloop technique was successfully used to close the mucosal entry at the end of POEM. The outcome was uneventful with conservative measures without any further clinical consequences.

All patients started drinking the following day after POEM and were able to return to normal activities within a week after the procedure.

Two octogenarians, a 79-year-old male with 45 years history of type III achalasia and sigmoid megaesophagus, who was previously treated by multiple balloon dilatations, and a 92-year-old female with 12 years history of type III achalasia and multiple episodes of aspiration pneumonia, who was also previously treated by dilatations, received bilateral POEM in order to finally solve the problem (Figure 2).
Both the patients had serious contraindications for surgery due to severe comorbidities, while all previous therapies failed. POEM was virtually the only realistic therapeutic option in these cases, which finally gave long-term excellent solution.

During follow-up, 6 months to 3 years after POEM, the great majority of patients $(90 \%)$ showed total clinical improvement (Eckardt score 0-1 after POEM), while one 79-year-old female, with sigmoid megaesophagus who underwent anterior POEM, showed moderate improvement of her dysphagia (Eckardt score: 3 after POEM), however, sufficient to maintain adequate nutrition for 3 years post-POEM.

Moreover, mild erosive esophagitis stage I (Los Angeles classification) showed three patients after POEM (15\%) successfully managed by proton pump inhibitor (PPI) on demand. No other short- or long-term complications related to POEM were reported, and all our patients were able to enjoy a satisfactory quality of life.

\section{Discussion}

The results of the present study were comparable to international standards, ${ }^{8}$ in terms of safety and efficacy of the POEM 
technique, while there was no restriction on the application of POEM regarding age or type of achalasia.

POEM is performed during endoscopy, and it is relatively painless and bloodless procedure without scar and short hospitalization time (1-3 days), while recovery time is also short, patients mobilized the same day and directly return to all of their daily activities within a few days.

One of the major advantages of POEM is the longest myotomy length of up to $25 \mathrm{~cm}^{3}$ (mean myotomy length in our study was more than $13 \mathrm{~cm}$ ), which is practically impossible with any other method.

Nevertheless, the oldest surgical Heller myotomy (1913), which is known for more than 100 years and also the more recent LHM are so aggressive and invasive, because they require both thoracotomy and abdominal incision to approach the esophagus, which practically make them prohibitive in terms of risk.

In contrast, the modern endoscopic myotomy approximates the diseased circular esophageal muscle, directly and easily through the submucosal tunnel, permitting safe and efficient long myotomy.

Another important advantage of POEM in contrast to all previous treatments is that it can be successfully applied without exceptions even to advanced types of achalasia, with megaesophagus, and also to debilitating elderly patients with high risk of surgery, such as in four of our patients.

POEM can also be successfully applied after failure of previous therapies, such as surgical myotomy or LHM or balloon dilatation. In these cases, POEM provides a longterm solution.

Four of our achalasia patients underwent successful POEM and solved their problems in long term, after failure of both previously repeated balloon dilatations and surgery. In these patients, POEM was practically the only realistic therapeutic option available.

Finally, repeat of the POEM technique (redo-POEM) was feasible and safe in short-time distance (within a month after first POEM) and significantly effective in two elderly patients (74-year-old male and 92-year-old female) with sigmoid esophagus and persistence of severe symptoms after previous otherwise successful anterior POEM.

These patients successfully underwent posterior POEM, discharged 1-day post-POEM and showed excellent clinical improvement remained in long-term one to two years postPOEM (Figure 2).

Another important advantage of POEM is that after POEM no antireflux procedure is necessary, because during POEM the normal antireflux mechanisms (diaphragmatic crus and angle of His) remain intact due to internal access through the submucosal tunnel, as it is also proved by the very low incidence of gastroesophageal reflux disease, usually mild and well controlled by PPIs on demand, as in our study (15\%), which is in accordance with the international literature $(10 \%-20 \%){ }^{2}$

On the contrary, in surgical myotomy or LHM, the normal antireflux mechanisms were disrupted due to access to the esophageal muscles from outside, and so antireflux procedures are usually necessary, if not in all cases. Moreover, antireflux procedures may be related to difficult complications.

Although 20 cases are not too much, the rarity of achalasia with annual incidence approximately $0.03-1 / 100,000$ per year, in combination with the difficulties and particularities of our 20 cases (octogenarians, sigmoid type, end-stage achalasia with megaesophagus, failure of all other previous therapies, including surgical myotomy), and the successful outcome in long term with a follow-up of more than 2 years make our study interesting.

We reported low level of intraoperative and postoperative complications, which is also in accordance with other international data. Particularly, we faced no cases of severe infectious inflammatory complications, such as mediastinitis, systemic inflammatory response syndrome sepsis, and severe bleeding or strictures.

Up to now, 6 months to 3 years post POEM all patients are alive $(0 \%$ mortality) and asymptomatic with very good quality of life. In the majority of cases (90\%), symptoms were totally resolved. A possible explanation of such exciting results is that we followed the strict protocol of POEM, according to the initial detailed description by Inoue et al. ${ }^{1}$ This study is ongoing.

\section{Conclusion}

According to our excellent results and in view of exciting results and advantages of POEM in the international level, we consider POEM to be safe and effective therapy of choice for all types of esophageal achalasia and all other esophageal motility disorders. Substantially, POEM has been established worldwide and appears to have replaced all prior treatments of achalasia. Further experience and long-term follow-up are necessary and awaited.

\section{Acknowledgment}

The authors thank Haruhiro Inoue for POEM training and case consultation.

\section{Author contributions}

All authors contributed toward data analysis, drafting and revising the paper and agree to be accountable for all aspects of the work. 


\section{Disclosure}

All authors disclosed no financial relationships relevant to this publication. Eleni Damianos Eleftheriadou is a niece of Nikolas Eleftheriadis. The authors report no other conflicts of interest in this work.

\section{References}

1. Inoue H, Minami H, Kobayashi Y, et al. Peroral endoscopic myotomy (POEM) for esophageal achalasia. Endoscopy. 2010;42(4): 265-271.

2. Pandolfino JE, Kwiatek MA, Nealis T, Bulsiewicz W, Post J, Kahrilas PJ. Achalasia: a new clinically relevant classification by high-resolution manometry. Gastroenterology. 2008;135(5):1526-1533.

3. Shiwaku H, Inoue H, Beppu R, et al. Successful treatment of diffuse esophageal spasm by peroral endoscopic myotomy. Gastrointest Endosc. 2013;77(1):149-150.

4. Onimaru M, Inoue $\mathrm{H}$, Ikeda $\mathrm{H}$, et al. Greater curvature myotomy is a safe and effective modified technique in per-oral endoscopic myotomy (with videos). Gastrointest Endosc. 2015;81(6):1370-1377.
5. Eleftheriadis N, Protopapas A, Katsogridakis J, Hatzitolios AI. Successful peroral endoscopic myotomy for radical treatment of sigmoid-type esophageal achalasia by Greek gastroenterologists. Ann Gastroenterol. 2014;27(4):430-431.

6. Zhou PH, Li QL, Yao LQ, et al. Peroral endoscopic remyotomy for failed Heller myotomy: a prospective single-center study. Endoscopy. 2013;45(3):161-166.

7. Onimaru M, Inoue $\mathrm{H}$, Ikeda $\mathrm{H}$, et al. Peroral endoscopic myotomy is a viable option for failed surgical esophagocardiomyotomy instead of redo surgical Heller myotomy: a single center prospective study. $J \mathrm{Am}$ Coll Surg. 2013;217(4):598-605.

8. Stavropoulos SN, Modayil RJ, Friedel D, Savides T. The International Per Oral Endoscopic Myotomy Survey (IPOEMS): a snapshot of the global POEM experience. Surg Endosc. 2013;27(9):3322-3338.

9. Eleftheriadis $\mathrm{N}$, Inoue $\mathrm{H}$, Ikeda $\mathrm{H}$, et al. Training in peroral endoscopic myotomy (POEM) for esophageal achalasia. Ther Clin Risk Manag. 2012;8:329-342.

10. Inoue H, Tianle KM, Ikeda H, et al. Peroral endoscopic myotomy for esophageal achalasia: technique, indication, and outcomes. Thorac Surg Clin. 2011;21(4):519-525.
Therapeutics and Clinical Risk Management

\section{Publish your work in this journal}

Therapeutics and Clinical Risk Management is an international, peerreviewed journal of clinical therapeutics and risk management, focusing on concise rapid reporting of clinical studies in all therapeutic areas, outcomes, safety, and programs for the effective, safe, and sustained use of medicines. This journal is indexed on PubMed Central, CAS,

\section{Dovepress}

EMBase, Scopus and the Elsevier Bibliographic databases. The manuscript management system is completely online and includes a very quick and fair peer-review system, which is all easy to use. Visit $\mathrm{http}: / /$ www.dovepress.com/testimonials.php to read real quotes from published authors. 